\title{
Wettability of Ceramic Substrates by Silver Based Alloys
}

\author{
Z. Weltsch* AND A. LOVAS
}

Budapest University of Technology and Economics, Department of Automobiles and Vehicle Manufacturing Stoczek u. 4, H-1111 Budapest, Hungary

(Received December 11, 2012)

The temperature dependence of wettability (wetting angle, $\Theta(T)$ ) for Ag-based melts on graphite and $\mathrm{Al}_{2} \mathrm{O}_{3}$ substrates is compared. Typical alloying effects are found, as the Ag host metal is gradually replaced by various metallic elements. The essence of alloying lies in the change of the electron/atom (e/a) ratio. This ratio is also manifested in the shift of wetting angles on the same substrate. The effect is also supported by the calculations based on the rigid band model, and is also in qualitative agreement with the Hume-Rothery rules. Nevertheless, the effects are partially smeared by other (metallurgical) factors, like the interaction between the oxygen-alloying elements and by the graphite substrate-oxygen interaction. In contrast, such effects are not pronounced in the case of $\mathrm{Al}_{2} \mathrm{O}_{3}$ substrates. As a consequence, $\Theta(T)$ exhibits an opposite trend in the case of two substrates. Crossovers of the $\Theta(T)$ curves were often found. The positions of crossovers depend on the chemical character and concentration of solute atoms. Segregation and epitaxial texture formation after solidification were also observed in certain alloy drops, especially in high concentration range. This phenomenon is not yet explained in every detail.

DOI: $10.12693 /$ APhysPolA.124.78

PACS: $68.08 . \mathrm{Bc}$

\section{Introduction}

Soldering is the most popular joint technique in microelectronic and optoelectronic industries which technologies are widely used in the vehicle industry [1-4].

Ensuring good wetting conditions between the solder material and the metallic parts to be joined is of great importance in these processes [5-11]. The Ag based alloys are particularly promising in this respect especially, when the worldwide trends in the replacement of lead-thin based alloys are considered [12-15].

In the previous papers [16-19], the wetting ability between graphite substrates and the $\mathrm{Ag}-\mathrm{M}(\mathrm{M}=\mathrm{Cd}$, In $\mathrm{Sn}, \mathrm{Sb}$ ) melts were reported, determined by the sessile drop method. Systematic change was detected in the wetting angle, when the Ag host metal was replaced by these elements or, when the solute concentration was also altered.

The temperature dependence $\Theta(\Delta T)$ was compared and the results were interpreted on the basis of classic alloying effects, i.e. based on the change in electron concentration $(\mathrm{e} / \mathrm{a})$, which is also reflected in the shape of the appropriate phase diagrams.

In this paper the $\Theta(\Delta T)$ measured on graphite and $\mathrm{Al}_{2} \mathrm{O}_{3}$ substrates is compared. Correlation between the thermopower $(S)$ and the residual resistance (measured in bulk solid state) and the wetting conditions will be described in the outlined silver-based alloys.

\section{Experimental conditions}

Alloys were prepared from high purity (4N) Ag and Sn, using induction melting in quartz crucible under inert (Ar) atmosphere. The graphite substrate was prepared from high purity, porosity free base material. The substrates were mechanically polished to $R_{\mathrm{a}}=0.23 \pm$ $0.005 \mu \mathrm{m}, r=1.009 \pm 0.0005$, then the surface roughness

*corresponding author; e-mail: weltsch@kgtt.bme.hu was determined using a 3D laser profilometer (Rodenstock RM600 surface topography measurement system).

The wetting experiments were performed in home-made equipment developed for sessile drop measurements. The experiments are described in detail in Ref. [20]. The graphite substrate and the alloy pieces were positioned into the middle of furnace at ambient conditions. The pressure was then reduced to $0.1 \mathrm{~Pa}$ at room temperature in the chamber. The vacuum was replaced by a $10^{5} \mathrm{~Pa} 99.999 \% \mathrm{Ar}$ gas. This procedure was repeated 3 times. Subsequently, the temperature was raised to $1273 \mathrm{~K}$ using a heating rate of $4 \mathrm{~K} / \mathrm{s}$. Since only a small part of the gas chamber is heated, no pressure change could be detected in the chamber $\left(10^{5} \mathrm{~Pa}\right)$ during the measuring run, which was performed during the heating run at $1273,1323,1373,1423$, and $1473 \mathrm{~K}$, respectively.

The heating power has stopped around each measuring temperature. The equilibrium contact angle was stabilized within one minute [21]. Two minutes holding time was applied before the measurement.

The contact angle was determined by measuring directly the profile of the drop. Self-made automatic software in the Matlab environment is used for fixing and processing the data. Though the uncertainty of this software is below 1 degree, the total uncertainty of the measured values is higher: $\pm 3^{\circ}$.

At the end of the measuring process at $1473 \mathrm{~K}$, the furnace was switched off and cooled slowly to the ambient temperature (the whole cooling time is around $40 \mathrm{~min}$ ). Subsequently the furnace was opened and the solidified sample was removed.

The vapour pressure of the sample components is different. Consequently, the preferential evaporation during the wetting angle measurements cannot be excluded. This effect can be the source of error in the contact angle measurements, because the wetting angle also depends on the sample mass, as it is illustrated by Fig. 1. Avoiding this effect, control measurements were performed. 


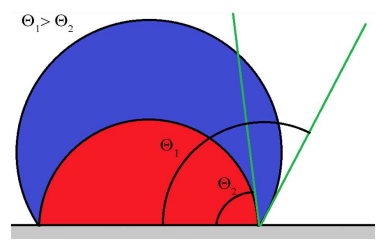

Fig. 1. The drop size effect.

The change of the samples mass.

TABLE

\begin{tabular}{c|c|c|c|c|c}
\hline \hline \multirow{2}{*}{ Sample } & \multicolumn{3}{|c|}{ Sample mass } & \multicolumn{2}{c}{ Alloying element mass } \\
\cline { 2 - 6 } & Before* & After* & Lost & Before* & Lost $^{* *}$ \\
\hline $\mathrm{Ag}$ & $0.2388 \mathrm{~g}$ & $0.2385 \mathrm{~g}$ & $0.13 \%$ & - & - \\
$\mathrm{Ag}_{95} \mathrm{Cd}_{5}$ & $0.2395 \mathrm{~g}$ & $0.2298 \mathrm{~g}$ & $4.05 \%$ & $0.01245 \mathrm{~g}$ & $80.32 \%$ \\
$\mathrm{Ag}_{95} \mathrm{In}_{5}$ & $0.2391 \mathrm{~g}$ & $0.2385 \mathrm{~g}$ & $0.26 \%$ & $0.0126 \mathrm{~g}$ & $7.14 \%$ \\
$\mathrm{Ag}_{95} \mathrm{Sn}_{5}$ & $0.2350 \mathrm{~g}$ & $0.2336 \mathrm{~g}$ & $0.6 \%$ & $0.0129 \mathrm{~g}$ & $13.18 \%$ \\
$\mathrm{Ag}_{95} \mathrm{Sb}_{5}$ & $0.2393 \mathrm{~g}$ & $0.2337 \mathrm{~g}$ & $2.34 \%$ & $0.0134 \mathrm{~g}$ & $44.02 \%$ \\
\hline
\end{tabular}

wetting angle measurement, ${ }^{* *}$ with the pure $\mathrm{Ag}$ correction

The weights of samples (drops) were compared before and after the measuring run. The results are summarized in Table. It is clear that a maximum weight loss can be detected in the case of $\mathrm{Ag}_{95} \mathrm{Cd}_{5}$ samples. As the vapour pressure was the highest in the case of $\mathrm{Ag}_{95} \mathrm{Cd}_{5}$, one can expect that the $\mathrm{Cd}$ loss contributes significantly to the resulting weight loss. It means that the $\mathrm{Cd}$ content of $\mathrm{AgCd}$ drop approaches to the pure Ag during the measuring run. Therefore exceptional behaviour of the $\Theta(T)$ curves is expected.

This anomaly can be the consequence of the significant continuous loss of the sample mass during the measuring run, but may also arise from the exceptional properties of $\mathrm{Cd}$. According to experiments (molten state) the interface area between the melt and substrate does not change in spite of the significant weight loss.

Scanning electron microscopy (SEM) investigations were carried out using a SEM type JEOL-JSM25-SIII, in secondary electron imaging mode. The constituent elements were analysed by a Bruker-Röntec EDS micro analyser system, attached to the SEM. The excitation parameters were $25 \mathrm{keV}$ beam energy and 50-200 pA beam current. The quantitative results were obtained from the measured spectra by a no-standard QUANTAX P/B ZAF correction program. The X-ray diffraction (XRD) measurements were performed with a Philips $\mathrm{X}$ 'Pert diffractometer and the profile fitting of the detected XRD patterns were carried out using the Pro'Fitt commercial program.

\section{The investigated alloys (metallurgical characterization)}

It is well known that several correlations exist between the bulk physical properties and the shape of phase diagram in metallic systems. On the other hand, it was also reported already in early papers that surface molar energy of liquid metals is in close connection with the heat of vaporization [22]. The correlation is similar with the melting point of the appropriate metals. It is also known that simple relation exists for the description of phase stability and the electron structure of simple alloys. The investigated alloys were selected on the basis of outline principles.

An additional motivation in the alloy selection was the simple solidification mechanism, i.e., the simple phase relation which is developed during the solidification.

All alloys (except the 10 at.\% Sb alloy) do form continuous series of solid solutions after solidification, i.e. only a single phase is formed from the molten state and no second phase precipitation occurs at low temperatures.

The position of solutes in the periodic table shows the increasing group number (Fig. 2).

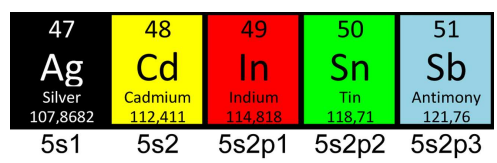

Fig. 2. The position of alloying elements in the periodic table.

The shape of the appropriate phase diagrams (details from the Ag-rich sides) are collected in Fig. 3. The common feature is the tendency of solid solution formation at the Ag-rich sides during the solidification. The solidified alloys are not homogeneous however, in atomic scale, as the solute segregation occurs. The degree of equilibrium segregation is reflected in the shape of the individual alloys. As the actual cooling rate is low (see the experimental description), this segregation can be regarded as "equilibrium segregation", so the concentration difference between the solid and the liquid difference does really represent the equilibrium distribution of the solute atoms in the solidified drop. The solubility limit can be explained on the basis of "electron phase" concept. Accordingly, the stability range of these solid solutions is governed by average valence electron concentration at the Fermi level of Ag. The upper limit of this concentration is around $(e / a \approx 1.36-1.59)$ for the stability limit of $\alpha$-solid solution in Ag. One can understood therefore that alloying (partial replacement of $\mathrm{Ag}$ by these elements in the sequence $\mathrm{Cd} \rightarrow \mathrm{In} \rightarrow \mathrm{Sn} \rightarrow$ Sb leads to the narrowing of the $\alpha$-phase region. The predictive power of this criterion is qualitatively supported by the well known Hume-Rothery solubility rules in these alloys $[23,24]$. The appropriate changes in the bulk physical properties in solid state have been reported already in previous papers $[25,26]$.

On the other hand it is clear, increasing difference in the slope of liquidus and solidus lines of these $\alpha$ solid solutions are in close connection with the mentioned degree of segregation, which reflects the degree of difference between chemical potential of solutes between the contacting phases at a given temperature.

The outlined tendencies can really be recognized from the comparison of the appropriate phase diagrams presented in Fig. 3.

In metals the surface energy, like other energy terms, has two main contributors: the lattice term and the elec- 


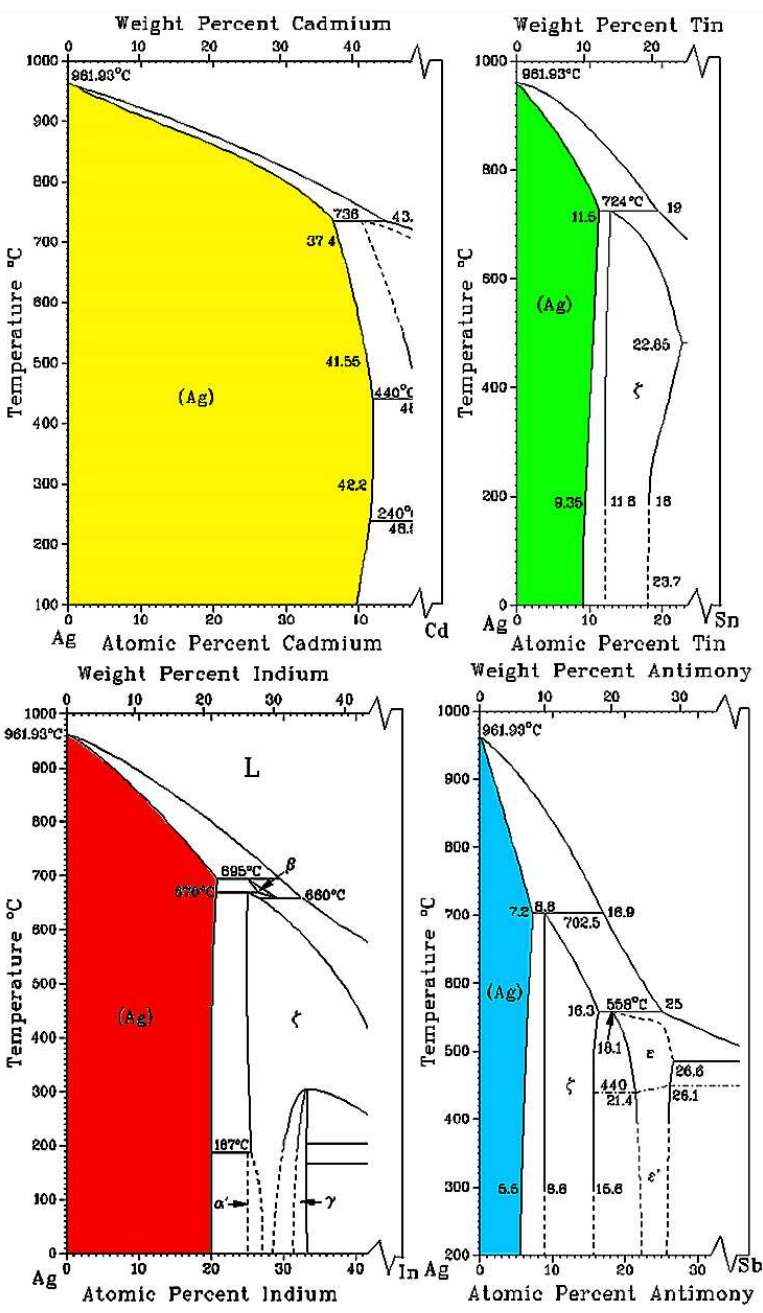

Fig. 3. The range of terminal $(\alpha)$-solid solutions in $\mathrm{Ag}-\mathrm{Cd}, \mathrm{Ag}-\mathrm{In}, \mathrm{Ag}-\mathrm{Sn}$, and $\mathrm{Ag}-\mathrm{Sb}$ systems [27].

tron gas term [19]. In normal and noble metals the cohesion is mainly represented by the electron gas. In the investigation of surface energy, the first question was whether the alloying effect (outlined before) can also be detected in the surface phenomena i.e. in the change of wetting of the melt at the surface of the same substrate. The substrate materials are (in the first approximation) inert (non-reactive) with respect to the molten Ag. The question is whether the change in the electron structure (caused by the metallic solute atoms) can also be detected in the surface energy. We will see that in other physical properties (mainly the electronic transport phenomena) this effect can be detected. A simple electronic structure calculations were also performed to calculate the surface energy change [28, 29].

The results of following calculations refer to liquid metals. The liquids are isotropic; therefore the surface tension is equal to the surface energy. Our consideration is based on the free electron model proposed by Ziman [30] for the description of resistance.
For surface energy calculation, the key is the modelling applied to the description of surface. In the frame of the Sommerfeld [31] concept, which is good enough for simple calculations, the liquid metal is represented by a potential well with depth of work function. The work function is high enough to apply an approximation of the infinitely deep well.

The metal is considered to have form of a slab. The slab is infinite in two directions: $x$ and $y$. In these directions periodic boundary $(k)$ conditions are used. In the third direction, the boundary condition is different. For reference we also use periodic condition. To obtain the surface energy we calculate the slab with infinite potential at both ends, that is, the wave function tends to zero at the ends. Starting relation is the energy $(E)$ of free electron gas of an effective electron mass $(m)$,

$$
E=2 \frac{\hbar^{2}}{2 m} \sum_{|k|<k_{\mathrm{F}}}\left(k_{x}^{2}+k_{y}^{2}+k_{z}^{2}\right),
$$

where $\hbar$ is the reduced Planck constant.

The summations are running through the quantum numbers, by the restriction, the energy is smaller than the Fermi energy, i.e. the modulus of wave number is smaller than the Fermi wave number. The factor 2 in the beginning of the formula counts the spins up and down. The slab has a periodic boundary condition in the directions $x$ and $y$, thus the summation in these directions can be replaced by integration. $A$ denotes the size of the surface, $A$ is parallel to the $x y$ plane, $\rho$ is the electron state density. In cylindrical coordinates

$$
E=\frac{\hbar^{2}}{m} \frac{A}{(2 \pi)^{2}} \sum_{\left|k_{z}\right|<k_{\mathrm{F}}} \int_{0}^{\sqrt{k_{\mathrm{F}}^{2}-k_{z}^{2}}} 2 \pi \rho\left(\rho^{2}+k_{z}^{2}\right) \mathrm{d} \rho,
$$

and after integration the next formula is obtained

$$
E=\frac{\hbar^{2}}{m} \frac{A}{2 \pi} \sum_{\left|k_{z}\right|<k_{\mathrm{F}}}\left(k_{\mathrm{F}}^{4}+k_{z}^{4}\right)
$$

If periodic boundary condition is applied in $z$ direction too, the reference system has no surface. The quantum number

$$
k_{z}=\frac{2 \pi}{L_{z}} n,
$$

in which the integer number $n$ runs over the positive and negative integers and zero fulfills the energy is smaller than the Fermi energy. If $n=0$, it is the lowest energy electronic state. All other states are twice degenerated energy terms. $L_{z}$ is the thickness of the slab, in this case it is the period of the boundary condition. If we have finite slab with surfaces, then

$$
k_{z}=\frac{\pi}{L_{z}} n,
$$

where $n$ is positive integer. In this case there is no $n=0$ states, and the terms are twice denser. The first one is lower than the first state of the reference system. The second one is just the same as the first (twice degenerated) one in the reference system. In every second term the system with surfaces has more energy caused by the wave number difference $\Delta k_{z}=\frac{\pi}{L_{z}} n$ between the two en- 
ergy values, given the energy of the two surfaces of the slab. The larger the thickness of the slab, the smaller the wave number difference. Therefore power expansion can be carried out. Thus the surface energy $(\gamma)$ is as follows:

$$
\gamma=\frac{\Delta E}{2 A}=\frac{\hbar^{2}}{m} \frac{1}{16 \pi} \sum_{0<k_{z}<k_{\mathrm{F}}} 4 k_{z}^{3} \Delta k_{z} .
$$

Now the summation over $k_{z}$ can be approximated by integration as usual

$$
\gamma=\frac{\hbar^{2}}{m} \frac{1}{16 \pi} \frac{L_{z}}{2 \pi} \int_{0}^{k_{\mathrm{F}}} 4 k_{z}^{3} \mathrm{~d} k_{z} \frac{\pi}{L_{z}}=\frac{\hbar^{2}}{m} \frac{1}{32 \pi} k_{\mathrm{F}}^{4} .
$$

The Fermi wave number is a common function of electron density, therefore our final formula is

$$
\gamma=\frac{\hbar^{2}}{m} \frac{1}{32 \pi}(3 \pi n)^{\frac{4}{3}}
$$

It is to conclude from the calculations that the electronic term for surface tension really depends on the electron density, i.e. on the electron/atom (e/a) ratio. This dependence is almost linear; therefore the successful experimental detection is expected in higher solute concentration only. In the frame of the experimental errors, the power $4 / 3$ is not distinguishable from the linear behavior.

\section{Sample control using electric resistance and thermopower measurements}

\subsection{Electric resistance measurements}

The investigated samples are (single phase) solutions both in liquid and solid state. Though the samples were chemically analyzed (energy dispersive spectroscopy EDAX) previously in the wetting experiments, some contamination may accrue during the long time procedure at high temperatures. The possible contaminations (traces of oxygen or C-atoms) arise from the surface of the substrate. The detection of these contaminations is difficult by EDAX measurements. Therefore, physical measurements were also introduced to control the possible change in the concentration (or the overall contamination) level before and after the wetting experiments. These methods are indirect, though they are sensitive enough for the detection of overall concentration change of solute components. Electrical resistivity and thermopower measurements are suitable for this purpose. It was reported in [32] that definite correlations exist between the shape of metallic phase diagrams and the composition dependence of electrical transport properties (conductivity) of the same alloy. The electrical resistivity rapidly increases in the range of solid solutions. This change is also specific to the chemical character of solute elements (valence and size-difference etc.). When some chemical interaction occurs between the solute atoms (either chemical reaction or precipitation) the resistance changes significantly without the overall concentration level. It is supposedly that specific resistance can supply information about the physical state of given components. This additional information is especially useful when the solute element is volatile, and the concentration may alter during the experiments.
Accordingly, the residual resistance was measured before and after the wetting experiments and this resistance changes were compared with the results of EDAX analysis.

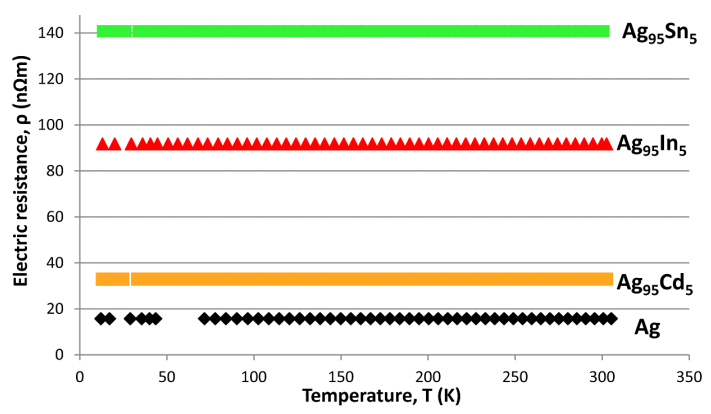

Fig. 4. The temperature dependence of electric resistance for 5 at.\% Ag-Cd, Ag- In, and Ag-Sn solid solutions.

As an example the temperature dependence of electrical resistivity is shown for pure $\mathrm{Ag}, \mathrm{AgCd}_{5}, \mathrm{AgIn}_{5}$ and $\mathrm{AgSn}_{5}$, in Fig. 4. The slope of $\rho(T)$ curves is negligible. Hence, in the $\rho(T)=0)$ limit, the Nordheim rule is fulfilled in the investigated alloys $[32,33]$.

According to $\rho_{\mathrm{r}}(x)=A x(1-x)$, where $\rho_{\mathrm{r}}$ is the residual resistivity, $x$ is the concentration of solute atoms. As the host metal is identical, $A$ is characteristic of the solute atoms. Hence, the trend in the resistivity increase reflects the increasing free electron concentration introduced by the host metal replacement.

\subsection{Thermopower $(S)$ measurements}

$S$ measurements were also introduced independent of the detection of sample-contaminations during the wetting experiments. The thermopower measurement is a suitable method for this purpose: this simple method is easy-realized even at around room temperature (low temperature is not necessary). In addition, the method is geometry-independent.

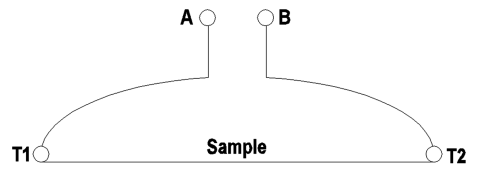

Fig. 5. Thermoelectric circuit.

The Seebeck coefficient is originally defined as the potential difference between two macroscopic metals being in contact according to the scheme in Fig. 5 and expressed by Eq. (9) [34, 35]:

$$
S_{A B}=\lim _{\Delta T \rightarrow 0} \frac{\Delta V}{\Delta T} .
$$

Instead of this differential form, an increasing temperature difference $(\Delta T)$ is applied according to the spirit of expression (10),

$$
S_{A B}=\int_{T_{1}}^{T_{2}}\left(S_{B}-S_{A}\right) \mathrm{d} T,
$$


where $T_{1}$ is the room temperature, $T_{2}$ is gradually changed from $T_{1}+1.5$ to $T_{1}+3$ values.

It was reported previously that $S(\Delta T)$ can supply information about the feature of the $\mathrm{M}-\mathrm{Cu}$ phase diagram, the phase transformations and also from the electronic structure of the investigated M metals [36, 37].

The $S(\Delta T)$ measurement is used for detecting the possible change in the electronic structure, caused by the dissolved alloying element. The dominant effect is the change in the electron density change caused by the solute atoms.

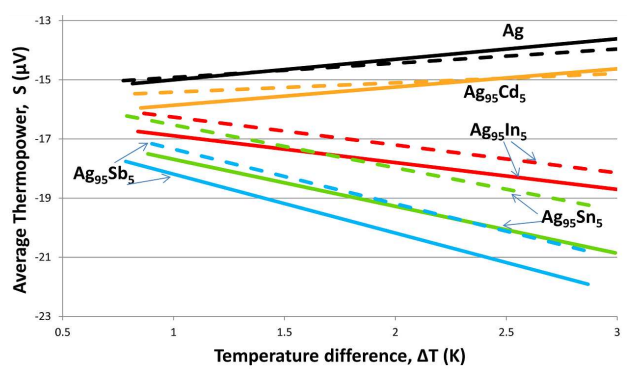

Fig. 6. The $S(\Delta T)$ plots for various Ag-based $\alpha$-solid solution, where the average electron concentration (e/a) changes only (marked with line before-, broken line after the wetting angle measurement).

In Fig. 6 the thermopower is plotted for $\mathrm{Ag}(\mathrm{Cd}$, In, $\mathrm{Sn}, \mathrm{Sb}$ ) alloys (solid solution, where the $\mathrm{Ag}$ is replaced by 5 at.\% solute). The temperature difference $\Delta T$ was gradually increased during the measuring run. Hence, each run represents several individual thermopower determinations. The shift of $S$ is characteristic for each alloy. The influence of $\mathrm{Cd}$ replacement is negligible. Even the sign of the slope is unchanged. This is expected from the small change in the e/a caused by the $\mathrm{Cd}$ solution. In contrast, both the sign and the shift of $S(\Delta T)$ becomes significant $S(\Delta T)$ when the $\mathrm{Ag}$ is replaced by $\mathrm{Sn}$ or $\mathrm{Sb}$.

The comparison of $S(\Delta T)$ runs is obtained from the initial and the remelted states (after the wetting measurements) and it is also informative. The small change arising from the contamination or in the stress level is also reflected in the curves (see $\mathrm{Ag}$ ). It was reported [38] that the deformation level has also an impact in the slope of $S(\Delta T)$. Comparing the $S(\Delta T)$ run obtained on the sample before and after the wetting measurements, the depletion of $\mathrm{Cd}$ content is obvious. The concentration decrease arises partly from the evaporation or it can be the consequence of deoxidation in molten state. As a whole, it is to conclude that shift of $S$ to negative direction or the pronounced change in the $S(\Delta T)$ slope is the consequence of the increasing group number difference (valence electron and electronegativity difference) between the Ag host and solute atoms.

\section{Wetting angle measurements}

The wetting angle was measured in wide temperature range. The wetting angles are illustrated as a function of the melt overheating (defined as $\Delta T=T_{\text {obs }}-T_{\text {liq }}$, where
$T_{\text {obs }}$ is the experimentally measured temperature, $T_{\text {liq }}$ is the liquidus temperature for the alloy at the investigated concentration). The favour of this illustration is obvious, as the slope of liquidus is very different (see Fig. 3, the phase diagrams). Comparing Figs. 7, 8 the first remarkable finding is the opposite temperature dependence of the $\Theta(T)$ slope obtained on the graphite and the $\mathrm{Al}_{2} \mathrm{O}_{3}$ substrates. It is also remarkable that the influence of the same solute atoms strongly depends on the melt superheat. The results are collected in Figs. 7, 8.

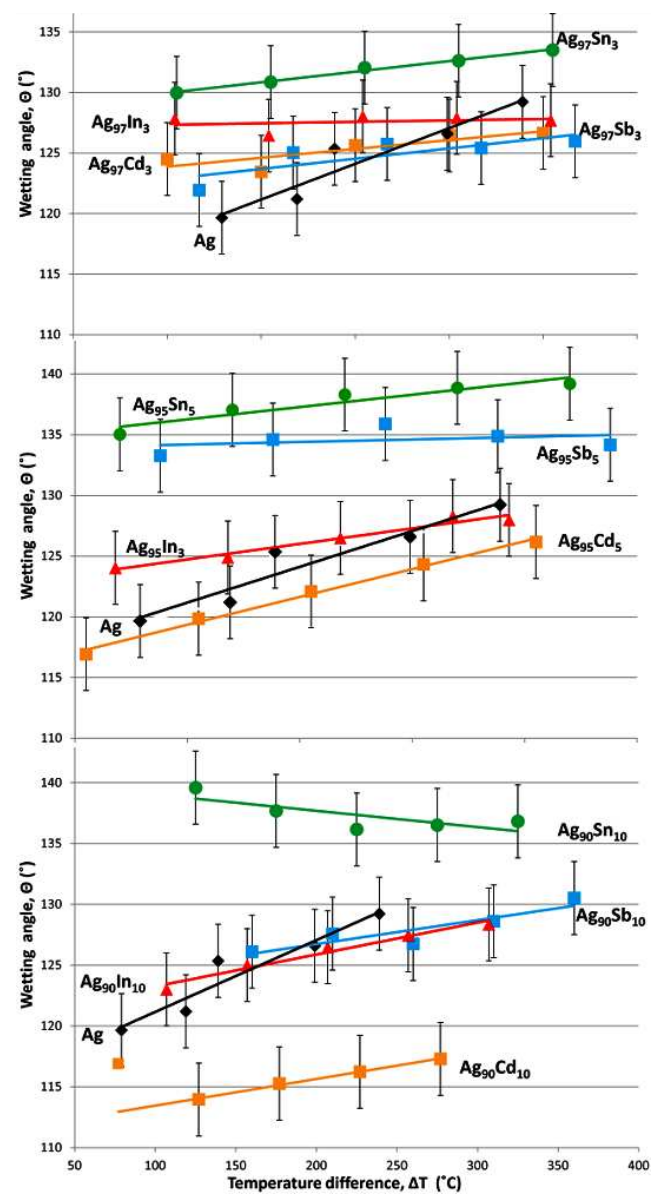

Fig. 7. Temperature dependence of the wetting angle of various $\mathrm{Ag}-\mathrm{Cd}, \mathrm{Ag}-\mathrm{In}, \mathrm{Ag}-\mathrm{Sn}, \mathrm{Ag}-\mathrm{Sb}$, and $\mathrm{Ag}$ liquids on graphite substrate.

As the phase-relations are very simple in these alloys (single phase nature of liquids and the solid solutions), one can suppose that the alloying induced by the electron concentration has a detectable impact not only in the stabilization of liquid state (melting point suppression) but also in the surface energy of the liquid drop. The magnitude of the wetting angle depends only indirectly on the total surface energy, which is composed from the drop/ gas and the drop/substrate interface components [39].

The summary of observations is the following: - The slope of $\Theta(T)$ for the pure $\mathrm{Ag}$ is positive on graphite, and negative on the $\mathrm{Al}_{2} \mathrm{O}_{3}$ substrates. This indicates the decreasing wetting ability of graphite, and 


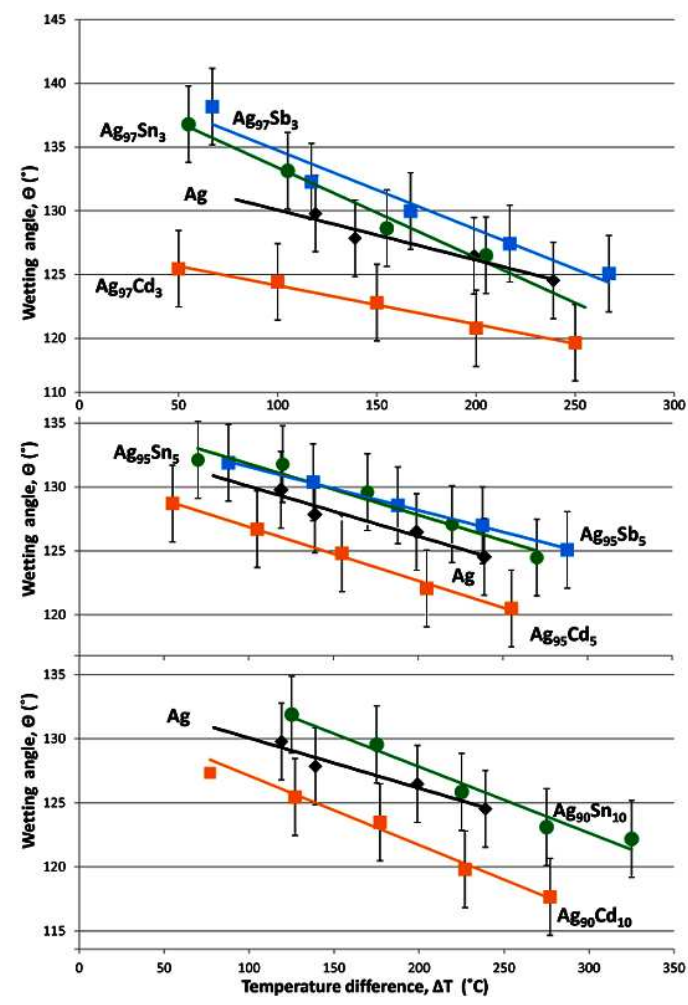

Fig. 8. Temperature dependence of the wetting angle of various $\mathrm{Ag}-\mathrm{Cd}, \mathrm{Ag}-\mathrm{In}, \mathrm{Ag}-\mathrm{Sn}, \mathrm{Ag}-\mathrm{Sb}$, and $\mathrm{Ag}$ liquids on $\mathrm{Al}_{2} \mathrm{O}_{3}$ substrate.

the increase of $\mathrm{Al}_{2} \mathrm{O}_{3}$ versus the melt superheat, i.e. the $\mathrm{Ag} /$ graphite interphase formation is energetically unfavorable in the same atmospheric circumstances, as the temperature increases (the interfacial energy increases) [22].

- The influence of alloying elements on $\Theta(T)$ is also opposite in the case of two substrates. The shift (at a given superheating) increases with the solute concentration, as it is expected from the residual resistivity measurements (see Fig. 9).

- Crossovers of the $\Theta(T)$ curves are often observed.

An influence of $\mathrm{Cd}$ addition is exceptional: generally opposite to that found in the case of other substituents ( $\Theta$ decreases compared to the pure $\mathrm{Ag}$ ). The lowering tendency is also typical on $\mathrm{Al}_{2} \mathrm{O}_{3}$ substrates. This general observation probably arises from the intrinsic property of $\mathrm{Cd}$ metal (exceptional high vapour pressure) [38].

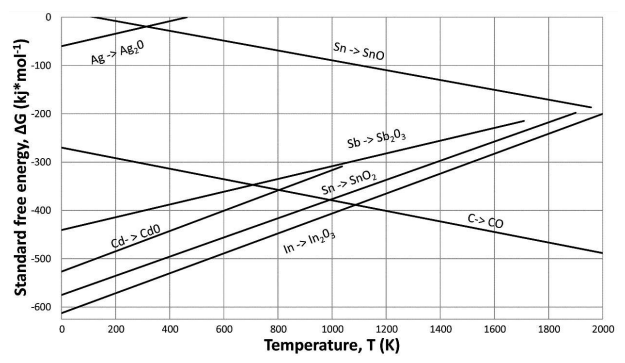

Fig. 9. Ellingham of the investigated systems [40].
The most remarkable finding obtained by the presented experiments is the marked difference between the slope of the $\Theta(T)$ belonging to the individual solute elements. This is not expected solely on the basis of e/a change, which predicts continuous bulk property change due to the also continuous electron density change around the Fermi level. This energy level should be insensitive to $\Delta T=200-300 \mathrm{~K}$ change beyond the melting temperature. This variation hints to participation of additional (mainly metallurgical) factors in the investigated wetting phenomena.

The compositional effects (arising from the electron density change, especially at low solute concentrations) are often smeared by the intervention of these factors. The O-traces may have primary importance in this respect. Probably the melt-substrate interaction has a significant impact. The O-contamination arises partially from the inner surface of structural elements of vacuum-chamber, which is covered by adsorbed oxygen molecules. As the temperature of the chamber does not increase the dissolved oxygen in the $\mathrm{Ag}(\mathrm{M})$ melt-drop seems to dominate. Based on this, the opposite slope of $\Theta(T)$ curves obtained on graphite and $\mathrm{Al}_{2} \mathrm{O}_{3}$ substrates can also be understood.

Qualitative explanation of the opposite trends observed on the investigated ceramic materials seems to be connected with the different reactivity of the two ceramics to the $\mathrm{O}$ traces, which can be appreciated from the Ellingham diagrams in Fig. 9. The selected data in this diagram refer to the affinity (and their temperature dependence) of the components to the oxygen which may be partly present in the environment, partly dissolved in the Ag itself (which is the dominant rough material from which the alloys were prepared). This dissolved $\mathrm{O}$ of $\mathrm{Ag}-\mathrm{O}$ liquid will react with the graphite substrate. According to the following reaction:

$$
\mathrm{Ag}(\mathrm{O})_{\text {liq }} \rightarrow \mathrm{Ag}+\mathrm{CO}_{\mathrm{g}}
$$

As the $T$-dependence of equilibrium constant for the reaction (11) is opposite than that for the metallic oxides formation (Fig. 9) the reaction is completed, when the temperature increases. Thus, further enrichment of $\mathrm{CO}$ molecules takes place at the melt-graphite interface. This reaction is dominant for the pure $\mathrm{Ag}$, therefore the slope of $\Theta(T)$ is the highest (rapid wettability decrease versus the increasing temperature).

When the liquid drop is such a solution, in which the reactivity of the dissolved $\mathrm{M}$ metal to the oxygen is higher than that for the $\mathrm{Ag}$ (see Fig. 9), interaction between the dissolved $\mathrm{O}$ and the $\mathrm{M}$ is developed in the melt according to

$$
\operatorname{Ag}(\mathrm{O})_{\text {liq }} \rightarrow \operatorname{Ag}(\mathrm{O}-\mathrm{M})_{\text {liq }}
$$

i.e. the metallic solute does also participate in the overall process. Hence, the activity of dissolved $\mathrm{O}$ is modified. At lower temperatures (near the liquidus) the reaction (11), at high temperatures the (12) reaction is the dominant (according to the spirit of the Ellingham diagram in Fig. 9). 
The crossovers between the $\Theta(T)_{\mathrm{Ag}}$ and $\Theta(T)_{\mathrm{Ag}-\mathrm{M}}$ curves can be understood based on the outlined interactions.

The opposite sign of $\Theta(T)$ in the case of $\mathrm{Al}_{2} \mathrm{O}_{3}$ can be imagined on the basis of substrate-degassing. As the temperature increases, O-desorption from the melt- $\mathrm{Al}_{2} \mathrm{O}_{3}$ interface takes place, which will dissolve in the Ag-melt. The O-enrichment in the Ag drop results in a surface tension lowering [38].

As a whole, two types of influences can be distinguished: One is powerful in the bulk liquid drop, near to the melting point of the drop, the second is more active at higher temperatures. Both arising from the opposite sign of the $T$ dependence equilibrium reaction are constants $\mathrm{C}-\mathrm{CO}$, and the $\mathrm{M}-\mathrm{MO}$ reactions, respectively.

This means that temperature dependence wetting ability is significantly modified by alloying elements. The existence of crossovers are probably associated with the different strengths of interactions between the $\mathrm{Ag}(\mathrm{M})-\mathrm{O}$ in the melt, as it is expected from the Ellingham diagrams.

The lowering tendency of $\Theta$ due to $\mathrm{Cd}$ addition observed both on the graphite and $\mathrm{Al}_{2} \mathrm{O}_{3}$ substrate is seen also in the case of $\mathrm{Al}_{2} \mathrm{O}_{3}$ substrates. This hints to the favour of melt-substrate interphase formation at the expense of melt-gas surface formation, when the $\mathrm{Cd}$ atoms are present in the melt drop. As it was mentioned above, the origin of this extraordinary behaviour can be attributed to the large heat of vaporization of $\mathrm{Cd}$. It was reported that surface tension of metallic melts at their melting points depends on the heat of vaporization [38].

However, the reason of opposite $\Theta(T)$ dependence of the wetting ability of graphite cannot be explained in every detail. The micro-porosity of two substrates is nearly the same (see Table). The indirect influence of packing density of crystal planes crossing the substrate-melt interface cannot be completely excluded [41]. The chemical absorption of O-traces - arising from the heating unit - may also contribute to the observed anomalies.

As it was mentioned, the behaviour of $\mathrm{Cd}$ solvent is exceptional in the investigated systems: $\Theta(T)$ usually decreases (especially at higher $\mathrm{Cd}$ content) which hints to increasing wettability due to the $\mathrm{Cd}$ addition. This tendency is particularly remarkable on the $\mathrm{Al}_{2} \mathrm{O}_{3}$ substrate. The wettability increase can be the indirect consequence of the high vapour pressure of $\mathrm{Cd}$, the overwhelming majority of $\mathrm{Cd}$ exhausted from the liquid during the wetting experiments (see Table). Consequently on the reaction suppressed, the liquid-C interface formation is energetically favourable.

Because of the exceptional high vapour pressure of $\mathrm{Cd}$, this reaction opens an additional discussion to the processes proposed by Eq. (12), which is going on recently.

\section{Possible correlations between the interphase segregation tendencies and the contact angle}

It was also observed that $\Theta(T)$ curves are composition dependent. Contrasts to the oxygen, the applied metallic solutes in question are not surface active in the $\mathrm{Ag}$ melt, so preferential surface enrichment is negligible. Consequently, the reason of drastic change (even in the sign of $\Theta(T)$ another phenomenon should be responsible (see $\mathrm{AgSn}_{10}$ in Fig. 7). In the range of low solute content $(3-5$ at.\%) the melts solidify in the form of single phase solid solutions, with fcc crystal structure (inherited from the Ag host).

In some cases however, when the slope of liquidus and solidus significantly differ, segregation tendencies can be developed, especially in the vicinity of surfaces, i.e. the drop is not perfectly homogeneous. Hence some uncontrolled chemical differences can be developed between the regions near to the melt-gas or melt-substrate interfaces. Such effects can be expected at high concentration. It is important to note that no detectable partitioning exists when the run of liquidus and solidus is very close $(\mathrm{Ag}-\mathrm{Cd})$. In contrast, the $\Theta(T)$ slope for $\mathrm{AgSn}_{10}$ and $\mathrm{AgGa}_{10}$ are very different (phase diagram $\mathrm{Ag}-\mathrm{Ga}[27]$ ). As an example, significant surface segregation can really be detected in $\mathrm{AgGa}_{10}$ alloy by the EDAX analysis of the cross-section of solidified AgGa drop (see Fig. 10).

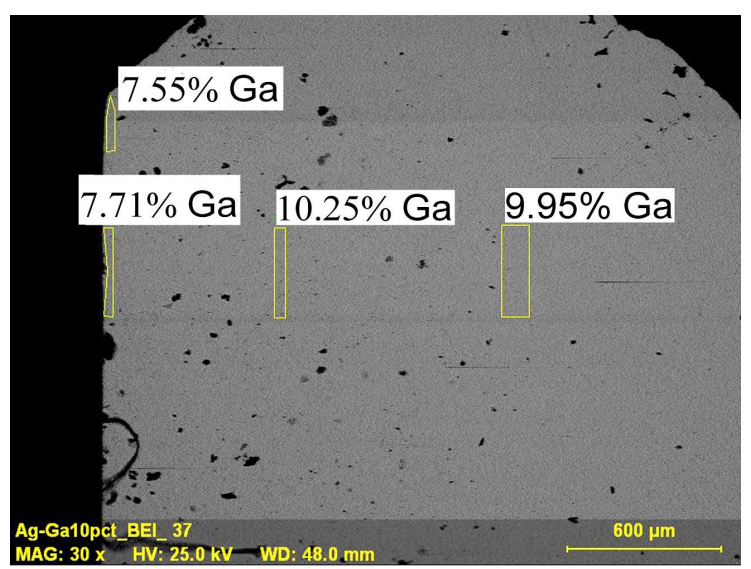

Fig. 10. The scanning electron microscopic picture from the cross-section of $\mathrm{AgGa}_{10}$ sample (1473 K), and the concentration values detected on the marked areas.

Based on the shape of phase diagrams, the highest segregation can really be expected in this case. This is also supported by the results of cross-sectional analyses (Fig. 10).

The Ga concentration is identified here by EDAX measurements near the substrate, comparing them with the inner side of the drop. The Ga-content is significantly lower near the substrate interface (around 7 at.\%), showing the depletion of alloying element in this region.

Texture formation in the solidified drop was observed near the graphite-drop interface, which seems to be in correlation between the degrees of melt superheated.

In Fig. 11 the XRD spectra are visible, taken from the graphite-side and inner part region of solidified $\mathrm{Ag}$ drop $2 a$ ( $1473 \mathrm{~K}$, inner part of the sample). The random distribution is the most intensive reflection very similar to the typical polycrystalline "powder" sample, where the (111) peak reflection is the strongest. 


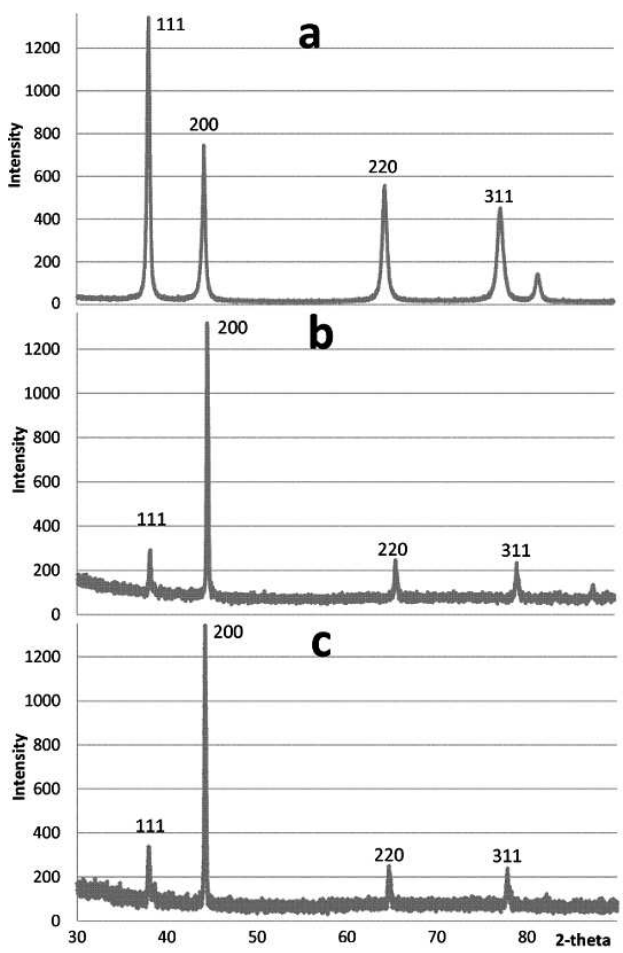

Fig. 11. XRD spectra measured on Ag sample solidified from $1473 \mathrm{~K}$ (a) middle of drop, (b) at the graphite interface, (c) sample cooled from $1273 \mathrm{~K}$.

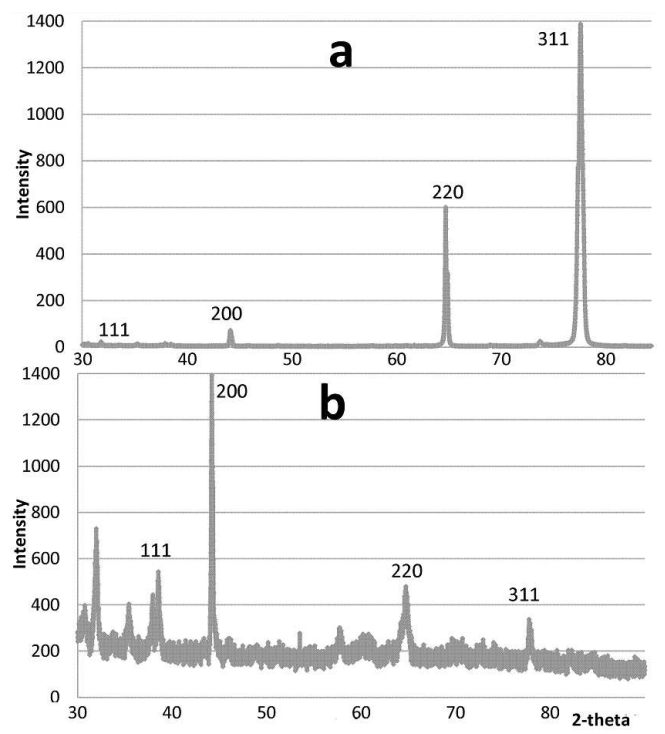

Fig. 12. The XRD spectra for Ag-Ga samples on the substrate side: (a) $1273 \mathrm{~K}$, (b) $1473 \mathrm{~K}$.

In contrast, the (200) reflection is the dominant near the drop-graphite interface showing, (100) planes are parallel to the graphite surface in the dominant fraction of grains. Comparing the intensity ratios obtained on samples (solidified from $1273 \mathrm{~K}$ and $1473 \mathrm{~K}$ ): $I(200) / I(111)=4.3$ and 8.16 , with the appropriate $(\Theta)$ values of $144^{\circ}$ and $132^{\circ}$ respectively, in agreement with the earlier observations.
In Fig. 12 the XRD spectra from $\mathrm{AgGa}_{10}$ sample (cooled from $1273 \mathrm{~K}$ and $1473 \mathrm{~K}$, respectively) are visible. The structure from $1273 \mathrm{~K}$ sample is similar to the pure Ag, i.e. the 200 reflection is the more pronounced reflection of the dominancy of 100 textures. The intensity ratio of reflections: $I(200) / I(111)=4.8$, the appropriate value is $139^{\circ}$.

In the sample with the smallest value (sample cooled from $1473 \mathrm{~K}$ ) the ratio of reflections' intensities differs completely from all other samples. The (311) and (200) reflections are the most intensive in contrast to the inner part of the sample, and the sample cooled from $1273 \mathrm{~K}$, where these intensities are extremely weak. It means that (311) and (220) planes are parallel to the graphite surface, i.e. the texture is more pronounced in the surface layer, causing the best wetting conditions, as the lowest value also supports $\left(118^{\circ}\right)$.

Though direct correlation between the slope of $\Theta(\Delta T)$ curves and texture formation is obviously not expected, one can speculate that some unspecified, high temperature chemical etching occurs at the interface beneath the melt drop, which may act as a catalytic surface-effect on the subsequent texture formation, when the solidification has started. Preferential, orientation dependent surface etching during high temperature vacuum-treatments is not unknown in the metallurgical praxis. Such effects can also occur beneath the melt drop. This effect is also more pronounced in the $\mathrm{Ag}$ and $\mathrm{Ag}-\mathrm{Ga}$ drops. The details of this effect (temperature dependence of texture formation), directed to the more detailed understanding of this texture formation should be further investigated in the future. In Fig. 12 the textured structure of the crystallites is the strongest at the interface in the $\mathrm{Ag}-\mathrm{Ga}$ $(1473 \mathrm{~K})$ sample. It can be seen that the (311) peak is the most intensive. In contrast, the crystal orientation in the interior of solidified drop is nearly random. Comparing the intensity values measured at $1273 \mathrm{~K}$ and $1473 \mathrm{~K}$ respectively, the outlined tendencies are further strengthened by the temperature increase as it can be drawn from Figs. 11, 12.

\section{Conclusions}

The temperature dependence of wetting angle is measured on graphite and $\mathrm{Al}_{2} \mathrm{O}_{3}$ substrates for various $\mathrm{Ag}-$ -based melts, in which the composition is systematically changed by the controlled replacements of host metal by metallic substituent.

- The shift and slope of $\Theta(T)$ compared to the pure $\mathrm{Ag}$ seems to be in correlation with the $S(T)$ curves and the magnitude of residual resistance - measured on the same alloy in solid state.

- The change of electrical resistance and the thermopower in the appropriate solidified alloys exhibits correlation with $\Delta \Theta$ in molten state. Hence the typical alloying effect postulated by the Hume-Rothery rules, as well as the change in $\mathrm{e} / \mathrm{a}$ (valence electron concentration) can be qualitatively recognized in the wetting properties of the investigated melts.

- The $a b$ initio calculation of electronic density change 
- due to the alloying does support the present experimental results.

- It was found that the temperature dependence of the wetting angle is opposite for the two substrate materials in the pure $\mathrm{Ag}$ and the investigated solid solutions.

- As a consequence, crossovers exist between the individual $\Theta(T)$ curves as a function of melt overheating. This means that at least two opposite factors exist in the temperature dependence of wetting ability of these alloy melts, indicating that the induced bulk alloying changes are often smeared by other metallurgical effects; like substrate melt interaction.

\section{Acknowledgments}

This work has been supported by the Hungarian Scientific Research Fund (OTKA) through grants No. K$-73690, \mathrm{~K}-109271$.

We want to express our thanks to Bence Tóth (Research Institute for Solid State Physics and Optics, Hungarian Academy of Sciences) for the support given during the measurement of the electric resistance.

\section{References}

[1] M. Judd, K. Brindley, Soldering in Electronics Assembly, 2nd ed., Newnes, Oxford (UK) 1999.

[2] J.H. Lau, Solder Joint Reliability of BGA, CSP, Flip Chip, and Fine Pitch SMT Assemblies, McGraw-Hill, New York 1997.

[3] K.N. Tu, Solder Joint Technology: Materials, Properties, and Reliability, Springer, New York 2007.

[4] C.-C. Fu, C.-C. Chen, J. Taiwan Inst. Chem. Eng. 42, 350 (2011).

[5] M. Erinç, P.J.G. Schreurs, G.Q. Zhang, M.G.D. Geers, J. Mater. Sci., Mater. Electron. 16, (10) 693 (2005).

[6] K.-L. Lin, C.-L. Shih, J. Electron. Mater. 32, 95 (2003).

[7] L.J. Matienzo, R.R. Schaffer, J. Mater. Sci. 26, 787 (1991).

[8] L.R. Garcia, W.R. Osório, L.C. Peixoto, A. Garcia, J. Electron. Mater. 38, 2405 (2009).

[9] T. Markovits, J. Takács, in: LANE 2004, Erlangen 2004, p. 263.

[10] A. Kroupa, A.T. Dinsdale, A. Watson, J. Vrestal, J. Vízdal, A. Zemanova, JOM 59, 20 (2007).

[11] G. Zeng, S. Xue, L. Zhang, L. Gao, Y. Hu, Z. Lai, J. Alloys Comp. 509, 7152 (2011).

[12] M. Abtew, G. Selvaduray, Mater. Sci. Eng. 27, 95 (2000).

[13] W.H. Zhong, Y.C. Chan, M.O. Alam, B.Y. Wu, J.F. Guan, J. Alloys Comp. 414, 123 (2006).

[14] C.M. Garner, V. Gupta, V. Bissessur, A. Kumar, R. Aspandiar, in: Proc. Third EPTC, Singapore, Eds. L.T. Beng, C. Lee, T.K. Chuan, Singapore 2000, p. 6.

[15] P. Casey, M. Pecht, in: Proc. Fourth Int. Symp. EMP, Taiwan 2002, p. 15.

[16] Z. Weltsch, A. Lovas, J. Machine Manufact. XLIX, 40 (2009).

[17] Z. Weltsch, A. Lovas, J. Takács, A. Cziráki, G. Tichy, A.L. Toth, L. Illés, Solid State Phenom. 159, 117 (2010).
[18] Z. Weltsch, A. Lovas, Mater. Sci. Forum 659, 109 (2010).

[19] Z. Weltsch, A. Lovas, G. Tichy, Z. Vandrus, Perner's Contact, Special Issue 2, Vol. VI, Pardubice 2011, p. 221.

[20] J. Hlinka, Z. Weltsch, J. Berzy, A. Szmejkál, Perner's Contact, Special Issue 2, Vol. VI, Pardubice 2011, p. 64 .

[21] Z. Weltsch, A. Lovas, J. Takács, A. Cziráki, A.L. Toth, G. Kaptay, Appl. Surf. Sci. 268, 52 (2013).

[22] B.C. Allen, in: Liquid Metals (Chemistry and Physics), Ed. S.Z. Beer, Marcel Dekker, New York 1972 , p. 165 .

[23] R.W. Cahn, in: Physical Metallurgy, Ed. T.B. Massalski, North-Holland Physics, Amsterdam 1983, Ch. 4.

[24] W. Hume-Rothery, in: Phase Stability in Metals and Alloys, Eds. P.S. Rudman, J. Stringer, R.I. Jaffee, McGraw-Hill, New York 1967.

[25] H. O'Neill, Hardness Measurements of Metals and Alloys, Chapman and Hall, London 1967.

[26] S. Flügge, Encyclopedia of Physics, Vol. XIX, Electrical Conductivity, Springer-Verlag, Berlin 1956, p. 208.

[27] Alloy Phase Diagrams Centre, ASM International, Materials Park $(\mathrm{OH}) 2006$.

[28] D.W. Hoffman, J.W. Cahn, Surf. Sci. 31, 368 (1972).

[29] D.W. Hoffman, J.W. Cahn, Acta Metallurg. 22, 1205 (1974).

[30] J.M. Ziman, Philos. Mag. 6, 1013 (1961).

[31] Sólyom Jenö, Fundamentals of the Physics of Solids II: Electronic Properties, ELTE Eötvös Kiadó, Budapest 2003, p. 602, (in Hungarian).

[32] The Structure and Properties of Materials, Vol. IV, Electronic Properties, Wiley, New York 1997.

[33] C. Kittel, Introduction to Solid State Physics, Múszaki Könyvkiadó, Budapest 1981, (in Hungarian).

[34] J.P. Fulton, M. Namkung, B. Wincheski, in: Rev. of Progress in Quantitative Nondestructive Evaluation, Eds. D.O. Thompson, D.E. Chimenti, Plenum Press, Brunswick (MA) 1993, p. 1611.

[35] G.S. Noals, J. Sharp, H.J. Goldsmid, Thermoelectrics. Basic Principles and New Materials, Developments, Springer, Berlin 2001.

[36] A. Szabo, A. Lovas, J. Machine Manufact. XLIX, 31 (2009).

[37] A. Szabó, Z. Weltsch, A. Lovas, Mater. Sci. Forum 659, 343 (2010).

[38] F.D. Richardson, Physical Chemistry of Melts in Metallurgy, Vol. 2, Academic Press, London 1974.

[39] G. Kaptay, J. Mater. Sci. 40, 2125 (2005) .

[40] DoITPoMS, University of Cambridge, http://www. doitpoms.ac.uk/tlplib/ellingham_diagrams .

[41] Z. Weltsch, A. Lovas, A. Cziráki, G. Tichy, A.L. Toth, Int. J. Appl. Mech. Eng. 15, 389 (2010). 